\title{
Trial of Oral Diuretics Prior to Discharge Is Not Associated With Improved Outcomes in Decompensated Heart Failure
}

\author{
Adeba Mohammad ${ }^{\mathrm{a}}$, Shuktika Nandkeolyar ${ }^{\mathrm{b}}$, Dennis Grewal ${ }^{\mathrm{b}}$, Antoine Sakr ${ }^{\mathrm{b}}$, \\ Ahmed Seliem $^{\mathrm{b}}$, Liset Stoletniy ${ }^{\mathrm{b}}$, Dmitry Abramov ${ }^{\mathrm{b}, \mathrm{c}}$
}

\begin{abstract}
Background: Current heart failure guidelines recommend transition of intravenous (IV) diuretics to oral $>24 \mathrm{~h}$ prior to hospital discharge. The aim of this study was to determine whether transition to oral diuretics prior to discharge in patients hospitalized with decompensated systolic heart failure (SHF) was associated with improved 30-day events.
\end{abstract}

Methods: This was a retrospective cohort study, in which adults admitted to the Loma Linda Medical Center for 3 - 14 days with a primary discharge diagnosis of acute on chronic SHF were included. Mortality data were obtained from the National Death Index, while readmission only to our facility was included. The $t$-test and Chisquare test were used for analyses.

Results: A total of 314 patients were studied. Patients who were managed with guideline-recommended trial of oral diuretics, and patients who continued to receive IV diuretics on the last full hospital day were overall similar in baseline characteristics. Patients who received oral diuretics on the day prior to discharge had longer length of stay, less weight loss, were discharged on lower diuretic doses (all $\mathrm{P}<$ 0.05 ), and had similar outcomes of 30-day readmission and 30-day hospitalization-free survival.

Conclusions: The transition to oral diuretics prior to discharge in patients with decompensated SHF was not associated with improved 30 -day outcomes. These results require validation in prospective trials but suggest that guideline recommendations regarding transitioning to oral diuretics prior to discharge may deserve re-evaluation.

Keywords: Diuretics; Readmissions; Heart failure; Hospitalization

Manuscript submitted April 5, 2021, accepted April 23, 2021

Published online July 9, 2021

aDepartment of Internal Medicine, Loma Linda University Health, 11234 Anderson St, Loma Linda, CA 92354, USA

bDepartment of Cardiology, Loma Linda University Health, 11234 Anderson St, Loma Linda, CA 92354, USA

${ }^{\mathrm{c}}$ Corresponding Author: Dmitry Abramov, Department of Cardiology, Loma Linda University Health, 11234 Anderson St, Loma Linda, CA 92354, USA. Email: dabramov@1lu.edu

doi: https://doi.org/10.14740/cr1265

\section{Introduction}

Heart failure (HF) affects 6.2 million people in the USA [1]. HF cost exceeded $\$ 30$ billion in 2012, and yearly costs are estimated to increase further to $\$ 70$ billion in $2030[2,3]$. The primary driver of HF cost is hospitalizations associated with HF exacerbation [4]. In addition to high healthcare cost, hospitalizations for $\mathrm{HF}$ exacerbation are associated with increased morbidity and mortality.

Volume overload is a primary cause of hospitalizations for patients with decompensated systolic HF (SHF) [5, 6]. Current HF guidelines recommend the treatment of volume overload with intravenous (IV) diuretics, with transition of IV diuretics to per os (PO) prior to hospital discharge [5-7]. The purpose of this recommendation is to determine whether patients are stable on an oral diuretic regimen prior to discharge, with the hope of preventing readmission, as readmissions have been associated with both a poor prognosis and increased costs $[8$, 9]. However, studies have shown that up to half of patients admitted for HF continue to receive IV diuretics on the day of discharge, with large variations among different hospitals [10-12].

While the American College of Cardiology (ACC)/American Heart Association (AHA) 2013 HF guidelines recommend a trial of oral diuretics prior to discharge, there is sparse evidence to support this practice and there is discrepancy among the current data available regarding the effects of transition from IV to PO diuretics in patients with acute decompensated SHF [11-13]. The aim of this study was to determine whether transition to oral diuretics for a full day prior to discharge in patients hospitalized with decompensated SHF was associated with improved outcomes, specifically 30 -day survival and readmissions.

\section{Materials and Methods}

This was a retrospective cohort study of all adults admitted to the Loma Linda Medical Center who were discharged with a primary diagnosis of acute on chronic SHF, or acute on chronic systolic and diastolic HF from 2013 to 2018. To be included, patients had to have received IV loop diuretic during the hospitalization. Patients with a hospital length of stay (LOS) of $<3$ 
days, $>14$ days, or those who died while hospitalized were excluded. Patients with LOS of $<3$ days were excluded because such patients would not be able to be transitioned to oral diuretics for a full day prior to discharge; and patients who were hospitalized for $>14$ days were excluded because this cohort likely represents a different patient population versus the average nationwide LOS for decompensated HF of 5.6 days [8]. If a patient was hospitalized more than once during the study period, only the first admission was included in the study. The study was approved by the hospital Institutional Review Board and is in compliance with the ethical standards of the institution.

Patients were divided into two groups based on whether they received any dose of IV diuretic (versus oral diuretics only) on the last full hospitalized day prior to discharge, as a full day of oral diuretic therapy would be suggested to ensure stability with oral diuretic administration. Electronic medical records were mined for relevant patient data. Data collected included baseline demographics, comorbid conditions, relevant laboratory values, vitals, weight changes, LOS, left ventricle ejection fraction, use of diuretics during the hospitalization (specifically furosemide, bumetanide, torsemide, and metolazone), and relevant medication use prior to admission and on discharge. Data on medication use prior to admission and post discharge focused specifically on dosing of loop diuretics, HF-approved beta blockers (carvedilol, bisoprolol, and metoprolol succinate), angiotensin system blockers, and aldosterone blockers. Mortality data were obtained from the National Death Index, while readmission for any cause only to our facility was included.

Hospitalization diuretic doses were calculated using IV equivalents of furosemide, unless otherwise indicated. For hospitalization diuretic dosing calculations, furosemide $40 \mathrm{mg}$ IV was equivalent to furosemide $80 \mathrm{mg}$ PO, bumetanide $1 \mathrm{mg}$ PO/IV, or torsemide $20 \mathrm{mg}$ PO. Diuretic doses were collected for day of admission (hospital day 0), hospital day 1, and last full day prior to discharge.

The primary outcomes of the study were 30-day all-cause readmission and 30-day readmission-free survival. Secondary outcomes included hospital LOS, weight changes during the hospitalization, differences between admission and discharge diuretic doses, and up-titration of HF therapy during the hospitalization.

\section{Statistical analysis}

Statistical analysis was conducted using $t$-test and Chi-square test where appropriate with level of significance of $<0.05$.

\section{Results}

A total of 314 patients were included in the study, after exclusion of 121 patients for LOS of $<3$ days and 35 patients for LOS of $>14$ days. Average age was $62.4 \pm 16.4$ years and $36 \%$ were female. Of the whole cohort, 173 patients received oral diuretics only on the last full day prior to discharge (group 1), while 141 patients received IV diuretics on their final full hospitalization day (group 2). Baseline characteristics, including age, sex, comorbid conditions, electrolytes, kidney function, pro-beta natriuretic peptide, blood pressure, pre-admission diuretic and dose, and left ventricle ejection fraction were similar between both groups (Table 1). The average LOS for the 314 patient cohort was $5.8 \pm 2.9$ days (while the average LOS for the cohort including excluded patients was $5.9 \pm 5.4$ days).

The diuretics and dosages administered to group 1 and group 2 were similar on hospital day 0 and day $1(\mathrm{P}=0.58$ and 0.60 , respectively) (Table 2 ). However, group 1 received a statistically significant lower diuretic dose on the last day of hospitalization, $66 \pm 42 \mathrm{mg}$ PO furosemide equivalents compared to $100 \pm 96 \mathrm{mg}$ IV furosemide equivalents for group $2(\mathrm{P}<$ $0.01)$. A higher percentage of patients in group 1 received metolazone compared to group 2 for days 0 and $1(\mathrm{P}<0.01$, and $\mathrm{P}<0.02$, respectively), but more patients in group 2 received metolazone compared to group 1 on the final hospitalization day $(\mathrm{P}<0.01)$. The administration of inotropes was similar for group 1 and group 2 (Table 2 ).

Patients in group 1 had longer average LOS (6.8 \pm 2.7 vs. $5.2 \pm 2.5$ days, $\mathrm{P}<0.01)$, but less weight loss compared to group 2 ( $2.1 \pm 4.2$ vs. $3.7 \pm 6.7, \mathrm{P}=0.01)$. Discharge electrolytes, creatinine and blood pressure were similar among group 1 and group 2 (Table 3). Additionally, group 1 was discharged on a lower diuretic dose (in PO furosemide dose equivalents) compared to group 2 (64 $\pm 44 \mathrm{mg}$ vs. $83 \pm 72 \mathrm{mg}, \mathrm{P}=0.01)$. More patients in group $1(23.8 \%)$ were discharged without oral loop diuretic compared to group $2(12.2 \%, \mathrm{P}<0.01)$. Further, more patients in group $1(25.0 \%)$ had their home loop diuretic dose decreased on discharge compared to group $2(15.1 \%, \mathrm{P}$ $=0.03$, Table 4). Despite longer LOS and transition to oral medications for at least a full day prior to discharge, there were no differences between groups in up-titration of guideline directed medication classes of beta blocker, angiotensin system blockers, or aldosterone blockers (Table 4).

There were no differences in the primary clinical endpoint post discharge between group 1 and group 2, with similar rates of 30-day readmission and 30-day hospitalization-free survival (Table 5).

\section{Discussion}

This analysis of diuretic use among patients hospitalized with a primary diagnosis of decompensated SHF demonstrates that patients transitioned to oral diuretics prior to discharge as suggested by guidelines had less weight loss and longer LOS compared to patients who continued to receive IV diuretics through their last full hospitalized day. Additionally, there was no difference in rates of HF therapy optimization and clinical outcomes, including 30-day readmission and 30-day readmission-free survival between the groups. These results suggest that recommendations about transition to oral diuretics prior to discharge among patients with decompensated HF deserve further evaluation.

Patients with decompensated SHF present to the hospital with symptoms of volume overload and congestion, including 
Table 1. Patient Characteristics

\begin{tabular}{|c|c|c|c|}
\hline Characteristics & $\begin{array}{l}\text { Oral diuretic on last full } \\
\text { day (group } 1, n=173 \text { ) }\end{array}$ & $\begin{array}{l}\text { IV diuretics on last full } \\
\text { day (group } 2, n=141 \text { ) }\end{array}$ & $P$ value \\
\hline Age (years) & $61.9(15.7)$ & $63.0(16.8)$ & 0.57 \\
\hline Sex (\%women) & $34.7 \%$ & $37.6 \%$ & 0.60 \\
\hline Diabetes mellitus (\%) & $23.1 \%$ & $24.8 \%$ & 0.73 \\
\hline Chronic obstructive pulmonary disease (\%) & $8.7 \%$ & $7.1 \%$ & 0.61 \\
\hline Chronic kidney disease (\%) & $17.3 \%$ & $17.0 \%$ & 0.94 \\
\hline Atrial fibrillation (\%) & $29.5 \%$ & $22.7 \%$ & 0.17 \\
\hline Admit sodium (mEq/L) & $137.3(5.4)$ & $137.6(5.06)$ & 0.65 \\
\hline Admit potassium $(\mathrm{mmol} / \mathrm{L})$ & $4.3(0.7)$ & $4.3(0.63)$ & 0.83 \\
\hline Admit creatinine $(\mathrm{mg} / \mathrm{dL})$ & $1.3(0.71)$ & $1.5(0.93)$ & 0.24 \\
\hline Admit furosemide PO dose equivalent (mg) & $71(49)$ & $76(61)$ & 0.54 \\
\hline Pre-admission diuretic & & & 0.77 \\
\hline$\%$ on furosemide & 88.9 & 90.2 & \\
\hline$\%$ on bumetanide & 5.7 & 3.9 & \\
\hline$\%$ on torsemide & 5.1 & 5.9 & \\
\hline
\end{tabular}

Data were presented as mean (standard deviation) or percentage (where indicated). IV: intravenous; PO: per os.

Table 2. In-Hospital Medication Administration

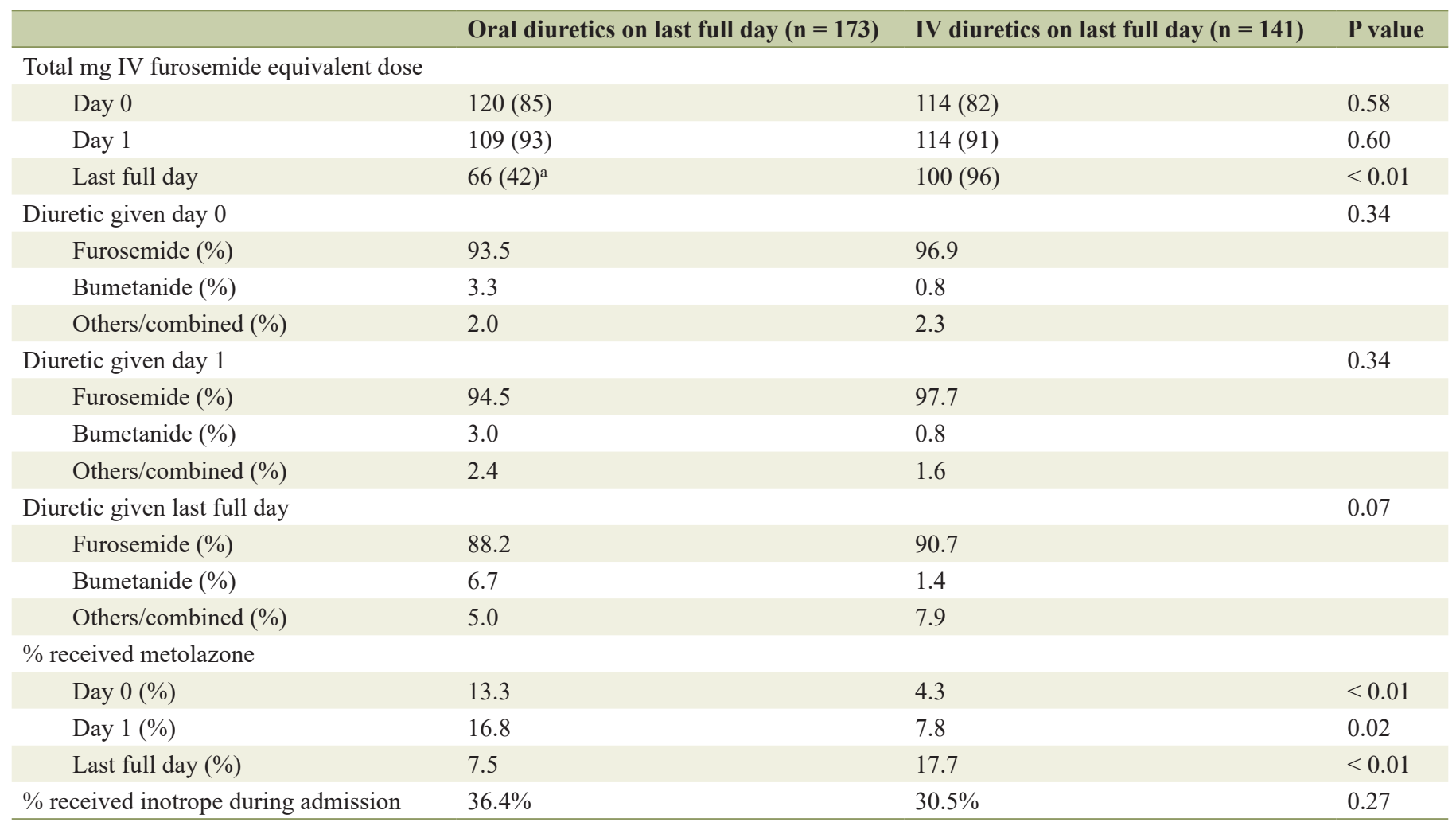

Data were presented as mean (standard deviation). alndicates oral diuretic dose. IV: intravenous. 
Table 3. Characteristics on Day of Discharge

\begin{tabular}{llll}
\hline Characteristics & Oral diuretics on last full day $(\mathbf{n}=\mathbf{1 7 3})$ & IV diuretics on last full day $(\mathbf{n}=\mathbf{1 4 1})$ & P value \\
\hline Discharge sodium $(\mathrm{mEq} / \mathrm{L})$ & $135.9(3.9)$ & $136.7(3.8)$ & 0.08 \\
Discharge potassium $(\mathrm{mmol} / \mathrm{L})$ & $4.2(0.4)$ & $4.1(0.4)$ & 0.06 \\
Discharge creatinine $(\mathrm{mg} / \mathrm{dL})$ & $1.4(0.7)$ & $1.5(0.8)$ & 0.22 \\
Discharge blood pressure $(\mathrm{mm} \mathrm{Hg})$ & $111(19) / 65(12)$ & $112(16) / 66(10)$ & $0.60 / 0.68$ \\
Average weight loss $(\mathrm{kg})$ & $2.1(4.2)$ & $3.7(6.7)$ & 0.01 \\
\hline
\end{tabular}

dyspnea and lower extremity edema $[5,6]$. The mainstay of treatment for patients with decompensated SHF is loop diuretics, with addition of non-loop diuretics as adjuncts $[14,15]$. While there is extensive data regarding initial IV diuretic use among patients with decompensated SHF, data are scarce regarding optimal diuretic administration prior to discharge, including possible benefits of transition from IV to oral diuretic and appropriate monitoring period for such transition. Current treatment guidelines recommend observation of patients on oral diuretics prior to hospital discharge $[5,6]$, which has been interpreted as at least a 24-h monitoring period [7], to be able to effectively gauge the effects of oral therapy. The primary reasoning behind recommendation to transition to oral diuretics is to verify the effectiveness of oral diuretic therapy, which may prevent readmission [7]. However, there is conflicting evidence regarding the efficacy of transition to oral diuretics, which could be used as basis for the guideline recommendation $[11-13,16]$. Prior observation studies demonstrated that up to a half of patients with decompensated HF continue to receive IV diuretics prior to discharge [10-12]. The current study, with $45 \%$ of patients remaining on IV diuretics on the day prior to discharge, adds to these prior publications.

The results of the current study are in accordance with prior preliminary descriptions of limited benefit of transition to oral diuretics prior to discharge, published only in abstract form $[12,13,16]$. A retrospective study of 285 patients found observation on oral loop diuretics for $>24 \mathrm{~h}$ prior to discharge was associated with longer hospital stays and no association with differences in 30-day outcome [12]. Another retrospective study of 240 patients found no difference in 30-day read-

Table 4. Discharge Medications

\begin{tabular}{|c|c|c|c|}
\hline $\begin{array}{l}\text { Medication class at goal dose or up titrated from admission } \\
\text { to discharge ( } \% \text { of patients })\end{array}$ & $\begin{array}{l}\text { Oral diuretics on last full day } \\
(\mathrm{n}=173)\end{array}$ & $\begin{array}{l}\text { IV diuretics on last full day } \\
(\mathrm{n}=141)\end{array}$ & P value \\
\hline Beta-blocker & $42.8 \%$ & $42.6 \%$ & 0.97 \\
\hline Spironolactone & $13.3 \%$ & $19.9 \%$ & 0.12 \\
\hline \multicolumn{4}{|l|}{ Loop diuretic dose adjustment compared to home loop diuretic dose } \\
\hline Decreased & $25.0 \%$ & $15.1 \%$ & 0.03 \\
\hline Discharge furosemide PO dose equivalent & $63(44)$ & $85(74)$ & $<0.01$ \\
\hline$\%$ discharged without diuretic & $23.8 \%$ & $12.2 \%$ & $<0.01$ \\
\hline Discharge diuretic & & & 0.97 \\
\hline
\end{tabular}

ACEI: angiotensin-converting enzyme inhibitor; PO: per os.

Table 5. Outcome Analysis

\begin{tabular}{llll}
\hline & Oral diuretic on last full day $(\mathbf{n}=\mathbf{1 7 3})$ & IV diuretics on last full day $(\mathbf{n}=\mathbf{1 4 1})$ & P value \\
\hline Length of stay (days) & $6.8(2.7)$ & $5.2(2.5)$ & $<0.01$ \\
30-day any readmission (\%) & $18.5 \%$ & $19.9 \%$ & 0.76 \\
30-day hospitalization-free survival (\%) & $80.4 \%$ & $78.0 \%$ & 0.61 \\
\hline
\end{tabular}

IV: intravenous. 
mission rates between patients who were transitioned to oral loop diuretics verses those who were not [13]. Finally, a larger study of 2,179 patients from the Get With the Guidelines data set similarly demonstrated higher LOS and no difference in 30-day readmission rates between groups transition to oral diuretics versus maintained on IV diuretics [16]. However, the current study conclusions differ from the published work by Laliberte et al, who conducted a retrospective study of 123 patient with acute decompensated HF, and noted that patients who were monitored on oral loop diuretics for under $24 \mathrm{~h}$ prior to discharge experienced significantly higher 30 -day HF readmission compared to those who were monitored on oral diuretics for greater than $24 \mathrm{~h} \mathrm{[11].} \mathrm{Several} \mathrm{differences} \mathrm{may} \mathrm{explain}$ the discordant findings between the study by Laliberte et al and current study. Although patient characteristics and study populations appear similar, differences in unmeasured patient characteristics, differences in clinician decision making regarding diuretic administration, and relatively small number of patients in both cohorts may contribute to divergent outcomes. Importantly, while both the study by Laliberte et al and the current study only collected readmission data from respective hospital systems (Liliberte et al from an 11-hospital network and the current study from the largest tertiary-care facility in the surrounding area), the 30-day readmission rates of the current cohort (approximately 20\%) more closely resemble national average compared to the rates from Laliberte et al (3.2\% in the patients transition to oral diuretics), which may increase the validity and generalizability of the current data.

Our study and the aforementioned retrospective studies show patients observed on oral diuretic $>24 \mathrm{~h}$ had longer hospital LOS. The primary contributor to cost of HF treatment in the USA is hospitalization [17]. Given the medical and financial burdens of HF in the USA, efforts to improve outcomes and reduce costs have received significant attention [4, 18-21]. Among these are efforts to reduce inpatient LOS and reduce 30-day readmissions. In the USA, there are approximately 1 million HF hospitalizations yearly [22]. Recommendations that attempt to balance inpatient LOS and reduction in readmission rates, including those regarding transition to oral diuretics before discharge, need to be evaluated in the context of effects on both metrics given potential for significant increases in total hospitalization days.

Many patients discharged for decompensated HF remain volume overloaded at the time of discharge [23, 24]; and guideline suggestions for transition to oral diuretics may need to be balanced with approaches to maximize inpatient time by focusing on attaining euvolemia with the more effective IV diuretics. While achievement of euvolemia with IV diuretics and transition to a trial of oral diuretics prior to discharge are not mutually exclusive, the financial and other burdens associated with the required increase in LOS to achieve the desired 24 $\mathrm{h}$ or greater observation period may deserve significant scrutiny. These data highlight the need for prospective randomized studies which measure costs, outcomes, and patient satisfaction to support guideline recommendation for observation of patients on oral diuretic therapy prior to discharge.

Additionally, it is unclear that an extra day of inpatient observation on oral diuretics in a hospital setting would be able to predict the effect of that regimen post discharge in the home en- vironment. While the extra hospitalized day can be used to optimize guideline-directed medical therapy, including facilitating initiation of newer agents including sacubitril-valsartan and sodium-glucose cotransport-2 inhibitors in selected patients, such approaches would require additional study and validation. Despite an increase in LOS, medication optimization was not different in the current cohort of patients monitored for an extra day on oral diuretics. Furthermore, more patients in the oral diuretic group were discharged home off oral diuretics, which may be associated with long-term adverse events [25].

\section{Study limitations}

This is a single-center study, with relatively small sample size of patients admitted to a tertiary care hospital, which may limit generalizability. Even though the two groups appear similarly matched based on baseline characteristics, hospital course, and discharge goal-directed medical therapy, there may be other confounders not accounted for, such as severity of HF, other medical factors associated with the hospitalization, or social barriers that may have contributed to LOS or medication decision making. Some data, including New York Functional Association class and other severity of HF, were not easily available in the electronic medical records. Only readmission to our facility was able to be tracked; however, given readmission rates that mirror (or only slightly underestimate) national averages, it is likely that the current data capture most readmissions in both groups. Only patients with SHF were evaluated in this study. Decisions regarding diuretic use were at the discretion of the treatment team, and rational for transition or lack of transition to oral diuretics prior to discharge is not available in this cohort. Metolazone was the formulary thiazide diuretic of choice at our facility, with other agents to augment diuresis not commonly used. Nevertheless, this study represents realworld clinical experience of inpatient clinical course and postdischarge outcomes among patients maintained versus transitioned off IV diuretics prior to discharge.

\section{Conclusions}

The transition to oral diuretics prior to discharge in patients with decompensated SHF was associated with less weight loss and increase in LOS without improved 30-day outcomes. These results require validation in prospective trials but suggest that guideline recommendations regarding transitioning to oral diuretics prior to discharge may deserve re-evaluation.

\section{Acknowledgments}

None to declare.

\section{Financial Disclosure}

The authors have no financial disclosure. 


\section{Conflict of Interest}

The authors have no conflict of interest to disclose.

\section{Informed Consent}

Informed consent was not obtained as this was a retrospective chart review and no patient identifiers are used.

\section{Author Contributions}

Dr. Adeba Mohammad (internal medicine resident) performed data analysis, created tables included in the manuscript, wrote the initial draft of the manuscript, and completed final formatting of submission documents. Dr. Shuktika Nandkeolyar (cardiology fellow) created the design of the study, performed data analysis, and provided critical edits to the final manuscript. Dr. Dennis Grewal (cardiology fellow), Dr. Antoine Sakr (cardiology heart failure attending), Dr. Ahmed Seliem (cardiology heart failure attending), and Dr. Liset Stoletniy (cardiology heart failure attending) provided critical edits to the final manuscript. Dr. Dmitry Abramov (cardiology heart failure attending) handled supervision, made substantial contribution to the conception of the study design, and provided substantial contribution to the final manuscript. He is the senior and corresponding author of the manuscript.

\section{Data Availability}

The data supporting the findings of this study are available from the corresponding author upon reasonable request.

\section{Abbreviations}

IV: intravenous; SHF: systolic heart failure; HF: heart failure; LOS: length of stay; PO: per os

\section{References}

1. Virani SS, Alonso A, Benjamin EJ, Bittencourt MS, Callaway CW, Carson AP, Chamberlain AM, et al. Heart disease and stroke statistics-2020 update: a report from the American Heart Association. Circulation. 2020;141(9):e139e596.

2. Benjamin EJ, Muntner P, Alonso A, Bittencourt MS, Callaway CW, Carson AP, Chamberlain AM, et al. Heart disease and stroke statistics-2019 update: a report from the American Heart Association. Circulation. 2019;139(10):e56e528.

3. Heidenreich PA, Albert NM, Allen LA, Bluemke DA, Butler J, Fonarow GC, Ikonomidis JS, et al. Forecasting the impact of heart failure in the United States: a policy statement from the American Heart Association. Circ
Heart Fail. 2013;6(3):606-619.

4. Urbich M, Globe G, Pantiri K, Heisen M, Bennison C, Wirtz HS, Di Tanna GL. A Systematic Review of Medical Costs Associated with Heart Failure in the USA (20142020). Pharmacoeconomics. 2020;38(11):1219-1236.

5. Yancy CW, Jessup M, Bozkurt B, Butler J, Casey DE, Jr., Drazner MH, Fonarow GC, et al. 2013 ACCF/AHA guideline for the management of heart failure: a report of the American College of Cardiology Foundation/American Heart Association Task Force on Practice Guidelines. J Am Coll Cardiol. 2013;62(16):e147-239.

6. Ponikowski P, Voors AA, Anker SD, Bueno H, Cleland JGF, Coats AJS, Falk V, et al. 2016 ESC Guidelines for the diagnosis and treatment of acute and chronic heart failure: The Task Force for the diagnosis and treatment of acute and chronic heart failure of the European Society of Cardiology (ESC)Developed with the special contribution of the Heart Failure Association (HFA) of the ESC. Eur Heart J. 2016;37(27):2129-2200.

7. Hollenberg SM, Warner Stevenson L, Ahmad T, Amin VJ, Bozkurt B, Butler J, Davis LL, et al. 2019 ACC expert consensus decision pathway on risk assessment, management, and clinical trajectory of patients hospitalized with heart failure: a report of the American College of Cardiology Solution Set Oversight Committee. J Am Coll Cardiol. 2019;74(15):1966-2011.

8. Kwok CS, Abramov D, Parwani P, Ghosh RK, Kittleson M, Ahmad FZ, Al Ayoubi F, et al. Cost of inpatient heart failure care and 30-day readmissions in the United States. Int J Cardiol. 2021;329:115-122.

9. Setoguchi S, Stevenson LW, Schneeweiss S. Repeated hospitalizations predict mortality in the community population with heart failure. Am Heart J. 2007;154(2):260266.

10. Fazel R, Strait KM, Bikdeli B, Dharmarajan K, Krumholz HM. Use of intravenous diuretics on day of discharge in adults hospitalized for heart failure. J Card Fail. 2014;20(9):706-707.

11. Laliberte B, Reed BN, Devabhakthuni S, Watson K, Ivaturi V, Liu T, Gottlieb SS. Observation of Patients Transitioned to an Oral Loop Diuretic Before Discharge and Risk of Readmission for Acute Decompensated Heart Failure. J Card Fail. 2017;23(10):746-752.

12. Schale S, Brambatti M, Hansen P, McCain J, Lin J, Greenberg B. Transitioning patients to oral diuretics 24 hours before discharge from heart failure hospitalization does not improve 30 day outcomes. Journal of Cardiac Failure. 2019;25(8):S61.

13. McCurdy FA, O'Dell DV, Susman J, Steele DJ, Paulman PM, Harper JL, Lacy NL. From library to discharge: a managing care student project. Fam Med. 2004;36 Suppl:S93-97.

14. Abdo AS. Hospital management of acute decompensated heart failure. Am J Med Sci. 2017;353(3):265-274.

15. Sinnenberg L, Givertz MM. Acute heart failure. Trends Cardiovasc Med. 2020;30(2):104-112.

16. Ingrassia Joseph, Marino Evan, Shivamurthy Poojita, Perucki William, Soucier Richard. Diuretics within 24 hours of discharge. Journal of the American College of Cardiol- 
ogy. 2014;63(12 Supplement):A532-A532.

17. Kilgore M, Patel HK, Kielhorn A, Maya JF, Sharma P. Economic burden of hospitalizations of medicare beneficiaries with heart failure. Risk Manag Healthc Policy. 2017;10:63-70.

18. Butler J, Djatche LM, Sawhney B, Chakladar S, Yang L, Brady JE, Yang M. Clinical and economic burden of chronic heart failure and reduced ejection fraction following a worsening heart failure event. Adv Ther. 2020;37(9):4015-4032.

19. Burns DJP, Arora J, Okunade O, Beltrame JF, BernardezPereira S, Crespo-Leiro MG, Filippatos GS, et al. International consortium for health outcomes measurement (ICHOM): standardized patient-centered outcomes measurement set for heart failure patients. JACC Heart Fail. 2020;8(3):212-222.

20. Wammes JJG, Auener S, van der Wees PJ, Tanke MAC, Bellersen L, Westert GP, Atsma F, et al. Characteristics and health care utilization among patients with chronic heart failure: a longitudinal claim database analysis. ESC Heart Fail. 2019;6(6):1243-1251.
21. Gupta A, Allen LA, Bhatt DL, Cox M, DeVore AD, Heidenreich PA, Hernandez AF, et al. Association of the hospital readmissions reduction program implementation with readmission and mortality outcomes in heart failure. JAMA Cardiol. 2018;3(1):44-53.

22. Jackson SL, Tong X, King RJ, Loustalot F, Hong Y, Ritchey MD. National Burden of Heart Failure Events in the United States, 2006 to 2014. Circ Heart Fail. 2018;11(12):e004873.

23. Maw AM, Lucas BP, Sirovich BE, Soni NJ. Dischargeready volume status in acute decompensated heart failure: a survey of hospitalists. J Community Hosp Intern Med Perspect. 2020;10(3):199-203.

24. Oehler A, Kent K, Davis J. Assessment of discharge readiness and readmission risk in patients with acute decompensated heart failure. Am J Cardiol. 2018;122(12):20862094.

25. Faselis C, Arundel C, Patel S, Lam PH, Gottlieb SS, Zile MR, Deedwania P, et al. Loop diuretic prescription and 30-day outcomes in older patients with heart failure. J Am Coll Cardiol. 2020;76(6):669-679. 